\title{
Design of a Teleoperation Scheme with a Wearable Master for Minimally Invasive Surgery
}

\author{
Gizem Ateş, Ronny Majani, and Mehmet İsmet Can Dede \\ Izmir Institute of Technology, Izmir, Turkey, \\ \{gizemates, candede\}@iyte.edu.tr \\ ronnymajani@gmail.com
}

\begin{abstract}
Minimally invasive surgery is increasingly being preferred over conventional surgery, however many problems still persist in longer surgeries such as pituitary surgeries, where surgeons are still required to hold an endoscope in their hand for prolonged periods of time. Many modern approaches have recently been proposed in literature to reduce the surgeon's effort. In this paper we extended upon these previous attempts and presented a promising solution; a real time teleoperation scheme with 3 different modes of operation, composed of a wearable ring system that captures and transmits voluntary hand motions over a wireless connection to a slave system. Accordingly, this slave system processes the received data to generate velocity demands for the robot endoscope controller. Finally, the feasibility of the proposed modes of operation are demonstrated and compared by measuring their learning curve and effort by running a set of training simulations on human subjects.
\end{abstract}

Keywords: teleoperation, assistive surgical robotics, human interface device, minimally invasive surgery

\section{Introduction}

Minimally invasive surgery (MIS) is a methodology that was developed to reduce the side effects of traditional open surgeries. However, it requires higher dexterity of the surgeons. To compensate for this increased requirement, the use of robot systems has become more preferable [5]. There are some commercial systems which are already in use today. We can group these surgical systems as autonomous and teleoperated robot systems. A review of autonomous medical robotic systems was conducted by Moustris et al. [3]. Furthermore, one of the earliest examples of teleoperated robotic surgical systems in endoscopic area was AESOP voice controlled endoscope manipulator [4]. We can further categorize the teleoperated surgical systems as surgeon off-site systems like the Da Vinci surgical robotic system [1] and on-site assistive robotic systems like Viky [6]. Da Vinci is a master/slave, system that consists of a surgeon's computer console to control the robotic tower that supports three or four robotic arms as the slave component. On the other hand, Viky offers a voice controlled "third hand" that handles the endoscope for the surgeon during a laparoscopic procedure.

This paper introduces a new on-site assistive surgical robot teleoperation system for minimally invasive pituitary surgeries. The next section explains the teleoperation 
system which is used for navigation of the endoscope by our novel master device. The operation modes of the teleoperation system is later explained which is followed by the description of the algorithm developed for these modes. Finally, the simulator constructed for user evaluations of the operation modes is introduced and the results are discussed by comparing the operation modes in terms of usability, learning curve and effort in use.

\section{Teleoperation System Overview}

The teleoperation system presented in this section allows the surgeons to control an endoscope remotely using their hand within a constrained environment (i.e. holding a tool that is partially inside a person's nose). This system is composed of three parts as; master, slave and communication channels which are depicted in Fig.1. The master consists of 2 parts:

- A ring that embeds a 6 DoF (Degree of Freedom) IMU (Inertial Measurement Unit) and Bluetooth module and is worn on the surgeon's index finger of the hand that will hold the non critical tools during the surgery. It transmits the angular velocity and acceleration of the surgeon's hand motions at a rate of $100 \mathrm{~Hz}$.

- A foot pedal that triggers the teleoperation scheme and sets the operation mode.

The slave consists of 3 parts:

- A microcontroller that implements the teleoperation scheme's algorithm described in section 4, in order to generate a set of velocity demands for the endoscope robot controller.

- Endoscope Robot Controller: a microcontroller that processes the generated endoscope velocity demands and utilizes motor drivers and signal processing to control the endoscope robot accordingly.

- Endoscope Robot: a manipulator with its joint actuators and sensors that handles an endoscope. This robot has 2 rotational DoF (pitch and yaw) and 1 translational DoF (dolly) around a remote center of motion [7].

There are two communication channels:

- Wireless: transmits the angular velocity and acceleration vectors measured by the ring to the microcontroller.

- Wired: transmits the pedal state to the microcontroller.

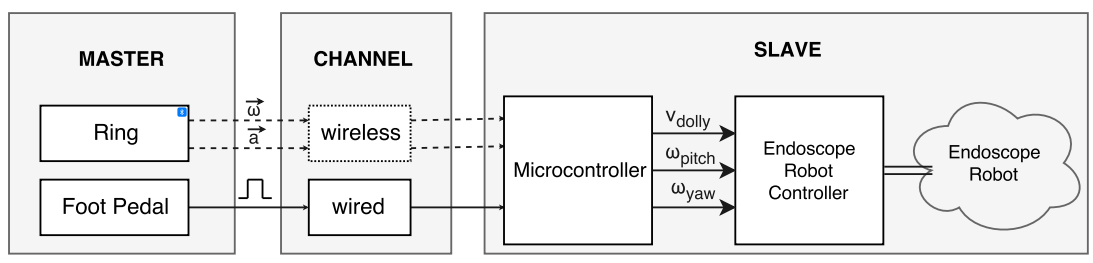

Fig. 1. Overview of the teleoperation system 
The teleoperation scheme renders hand motions by integrating the angular velocity that the ring transmits. It calculates the angular position difference since the foot pedal was pressed, and then maps these angles to the proportional velocity demands for the endoscope according to the current system state. This scheme is also known as the displacement to velocity teleoperation scheme as described in [2].

There are 3 system states: $O F F$, ROTATION, and DOLLY. In the $O F F$ state the pedal is released, the teleoperation scheme is inactive, and the angle accumulator is reset. In the ROTATION and DOLLY states, the pedal is pressed, and the teleoperation scheme is active. The active system state is assigned according to the way the surgeon chooses to press the foot pedal which is described in section 4 .

A visualization example of the angle accumulation of the ring's pitch motion and its mapping to a velocity demand for the endoscope's pitch motion is given in Fig.2.

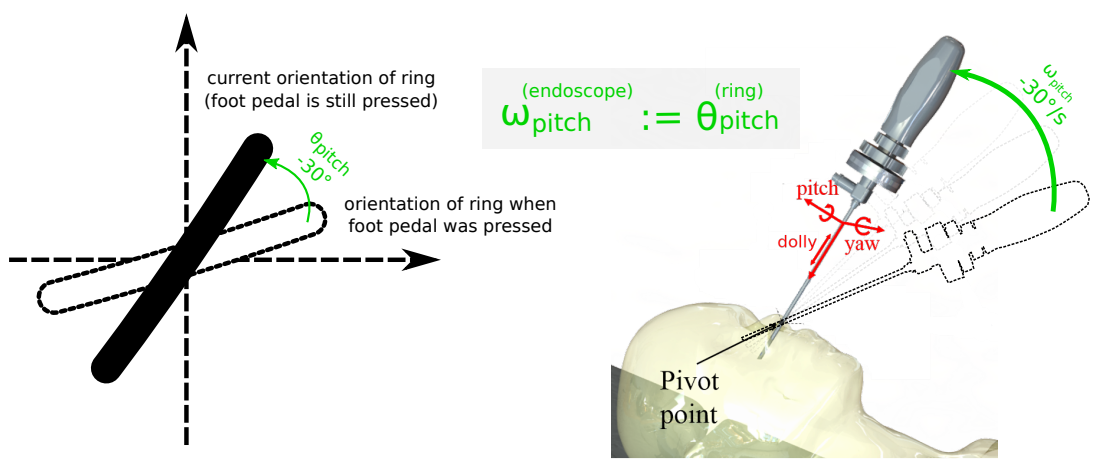

Fig. 2. Visual example of angle accumulation and its mapping to a velocity demand

\section{Operation Modes}

We propose a teleoperation scheme with 3 operation modes, two modes where the fingers are coupled to a tool (Coupled Modes), and one mode where the index finger is uncoupled (Uncoupled Mode).

\subsection{Coupled Modes}

In the two coupled modes, the surgeon is able to control the endoscope while maintaining their full grip around a tool. The ring's motions are performed by rotating the tool. The rotational motions are mapped in a way that it seems the direction the tool's tip motion is proportional to the direction the endoscope's tip motion. To allow for such motions, axis alignment is performed to compensate for the ring frame's misalignment with respect to the Earth's frame while the surgeon is holding the tool. Axis alignment will reorient the angular velocities measured by the ring so they are rendered in the Earth's frame.

The two variations of coupled mode differ in the mapping of the endoscope's dolly motion. The mapping of each mode's motions is described in Tables 1 and 2: 
- coupled 3D mode: when the system is in the DOLLY state, the ring's roll motion is mapped to the endoscope's dolly motion.

- coupled 2D mode: when the system is in the DOLLY state, the ring's pitch motion is mapped to the endoscope's dolly motion, meaning we controlling a 3 DoF Robot using only 2 DoF motion.

Table 1. Mapping of axis aligned ring motions to Table 2. Mapping of axis aligned ring motions to endoscope velocity demands for coupled mode endoscope velocity demands for coupled mode $3 \mathrm{D}$ $2 \mathrm{D}$

\begin{tabular}{|c|c|c|}
\hline mode & $\begin{array}{c}\text { aligned ring } \\
\text { motion }\end{array}$ & $\begin{array}{c}\text { endoscope velocity } \\
\text { demand }\end{array}$ \\
\hline \hline \multirow{2}{*}{ ROTATION } & $\theta_{\text {pitch }}$ & $\omega_{\text {pitch }}$ \\
\cline { 2 - 3 } & $\theta_{\text {roll }}$ & $\omega_{\text {yaw }}$ \\
\hline \hline DOLLY & $\theta_{\text {yaw }}$ & $v_{\text {dolly }}$ \\
\hline
\end{tabular}

\begin{tabular}{|c|c|c|}
\hline mode & $\begin{array}{c}\text { aligned ring } \\
\text { motion }\end{array}$ & $\begin{array}{c}\text { endoscope velocity } \\
\text { demand }\end{array}$ \\
\hline \hline \multirow{2}{*}{ ROTATION } & $\theta_{\text {pitch }}$ & $\omega_{\text {pitch }}$ \\
\cline { 2 - 3 } & $\theta_{\text {roll }}$ & $\omega_{\text {yaw }}$ \\
\hline \hline DOLLY & $\theta_{\text {pitch }}$ & $v_{\text {dolly }}$ \\
\hline
\end{tabular}

\subsection{Uncoupled Mode}

In this mode, no axis alignment is performed, meaning the teleoperation scheme is invariant to the ring frame's initial orientation. The surgeon depends on their index finger's motions to control the endoscope. They are rendered in the ring's own frame. Intuitively, the surgeon would be holding a tool with their hand except for the index finger which would be free to move when the surgeon wishes to control the endoscope. Also due to the physical difficulty of performing roll motion while the surgeon's hand is gripping a tool, the pitch motion of the ring is mapped to the dolly motion of the endoscope when the system is in the DOLLY state. The mapping of the ring's motions to the endoscope's motions are described in Table 3.

Table 3. Mapping of ring motions to endoscope velocity demand for uncoupled mode

\begin{tabular}{|c|c|c|}
\hline mode & $\begin{array}{c}\text { ring } \\
\text { motion }\end{array}$ & $\begin{array}{c}\text { endoscope velocity } \\
\text { demand }\end{array}$ \\
\hline \hline \multirow{2}{*}{ ROTATION } & $\theta_{\text {pitch }}$ & $\omega_{\text {pitch }}$ \\
\cline { 2 - 3 } & $\theta_{\text {roll }}$ & $\omega_{\text {yaw }}$ \\
\hline \hline DOLLY & $\theta_{\text {pitch }}$ & $v_{\text {dolly }}$ \\
\hline
\end{tabular}

\section{Teleoperation Scheme's Algorithm}

The teleoperation scheme's algorithm is visualized as a linked chain of nodes in Fig.3, each with its own functionality. In this system, there are two sources of input: the ring worn by the surgeon, and a foot pedal. The ring transmits 6 DoF information: 3 DoF 
angular velocity vector $\vec{\omega}$, and 3 DoF acceleration vector $\vec{a}$. The foot pedal works as a binary trigger with two states: pressed, and released. Axis alignment is only done in the coupled modes. In uncoupled mode, the acceleration vector $\vec{a}$ is not used, and the angular velocity $\vec{\omega}$ is fed directly to the angle accumulator. At the end of the chain, the output of the teleoperation scheme is passed to the endoscope robot controller.

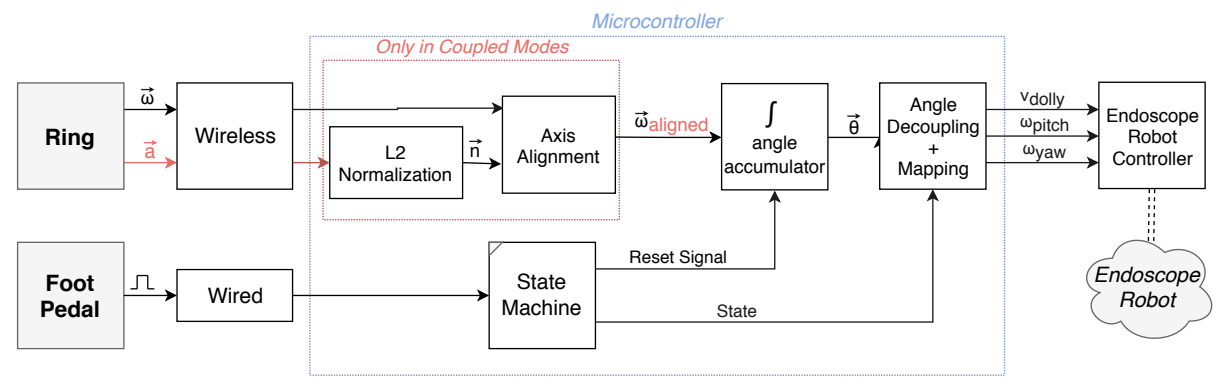

Fig. 3. An overview of the teleoperation scheme.

Axis Alignment We compensate for the misalignment of the ring's vertical axis and the Earth frame's vertical axis (determined by the unit vector $\vec{n}$ of the ring's accelerometer data) by using a transformation matrix $\widehat{C}^{(e, r)}$ composed of two Euler rotations to transform the angular velocities measured in the ring frame $\mathscr{F}_{r}$ to their equivalent angular velocities in the Earth's frame $\mathscr{F}_{e}$.

Given the raw accelerometer acceleration vector $\vec{d}$ as input, we first calculate the gravity direction in the ring frame as a unit vector $\vec{n}$ :

$$
\vec{n}=\frac{\vec{a}}{\|\vec{a}\|}=n_{x}{\overrightarrow{u_{x}}}^{(r)}+n_{y}{\overrightarrow{u_{y}}}^{(r)}+n_{z}{\overrightarrow{u_{z}}}^{(r)}
$$

This unit vector $\vec{n}$ is used in order to determine how much the ring is oriented with respect to the Earth's frame. Since the gravity vector is defined as $\vec{g}=g \cdot{\overrightarrow{u_{z}}}^{(e)}$ in Earth's frame, we cannot determine the orientation about $\vec{u}_{z}^{(e)}$. Assuming the ring is oriented with an Euler Y-X sequence by $\alpha$ and $\beta$ amount of rotations respectively, the transformation matrix $\widehat{C}^{(e, r)}$ from ring's frame to Earth's frame is evaluated as follows:

$$
\widehat{C}^{(e, r)}=e^{\tilde{u}_{y} \alpha} e^{\tilde{u}_{x} \beta}
$$

where, its $\alpha$ and $\beta$ values can be determined by solving the following equation:

$$
\bar{n}^{(r)}=\widehat{C}^{(r, e)} \bar{u}_{z}
$$

Then we calculate the aligned angular velocity $\vec{\omega}_{\text {aligned }}$ which is the input to the accumulator node in the Coupled Modes, with the following formula:

$$
\left.\left\{\vec{\omega}_{\text {aligned }}\right\}\right|_{\mathscr{F}_{e}} \triangleq \bar{\omega}^{(e)}=\hat{C}^{(e, r)} \cdot \bar{\omega}^{(r)}
$$


Angle Accumulator The angle accumulator converts the raw IMU gyroscope data (angular velocity $\vec{\omega}$ ) to the angle displacement which will be processed to be a velocity demand. It is active and accumulating when the teleoperation scheme is active, and is reset and stopped when the teleoperation scheme is deactivated. The angle accumulator is mathematically modeled in (5), with $K_{i}$ being the scaling constants, and $\sigma$ being the activation value and is set to 1 when the teleoperation scheme is active, and 0 otherwise.

$$
\theta_{i}=s a t\left[K_{i} \cdot \sigma \int \omega_{i}^{(j)} d t\right] ; \quad i=\text { pitch,yaw, roll; } \quad j= \begin{cases}r & \text { in Uncoupled Mode } \\ e & \text { in Coupled Modes }\end{cases}
$$

State Machine The state machine is responsible for managing the system state and its transitions based on the foot pedal press states. The transition of the system's states are shown in Fig.4 and described as follows:

- $O F F$ : set when the pedal is released. The angle accumulator is reset and stopped, and the teleoperation scheme is inactive.

- ROTATION: set when the foot pedal is pressed and held down longer than a predefined time called the double click tolerance $\tau_{\text {dolly }}$. Only motions of the ring mapped to the rotational motions of the endoscope are processed.

- DOLLY: set when the foot pedal is pressed 2 consecutive times, where the time elapsed between the two pedal presses is no longer than $\tau_{d o l l y}$. Only the motion of the ring mapped to the dolly motion of the endoscope is processed.

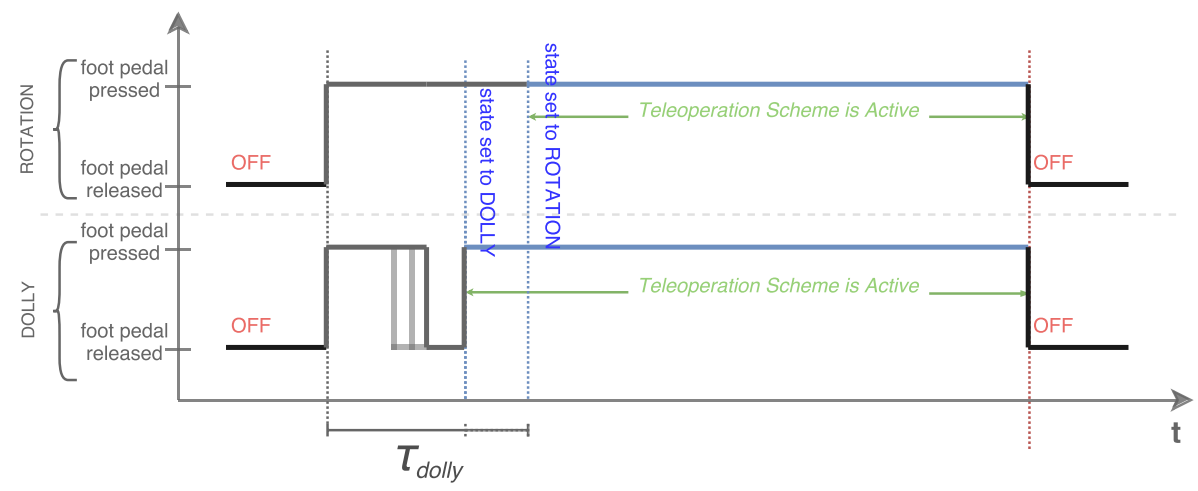

Fig. 4. Visualization of system state transitions.

Decoupling and Mapping The final step of the scheme is converting and mapping the accumulated angle displacement to appropriate velocity demands for the endoscope robot controller according to the current system state. The mapping is fully dependent on the mode of operation and is described in their relevant subsections (3.1 and 3.2).

\section{Feasibility Study}

To measure, assess, and compare the feasibility of the proposed operation modes, a training simulator is developed. The simulation environment is composed of: 
- A simulation software running on a computer, that consists of a 2D virtual environment that will mock the endoscope controller, endoscope robot and its environment as shown in Fig.5.

- In this environment, there are pre-specified circles at various coordinates with varying radii, and a special circle that the human subject controls, which we call the "tool". In this environment, endoscope robot's pitch and yaw demands are processed as vertical and horizontal translations of the tool respectively, while the dolly demand is processed as the increase/decrease of tool's radius,

- A 3D printed human skull model, with a soft rubber nose, and an aspirator tool that the test subjects hold with its tip positioned inside the nose,

- Ring, Foot Pedal, and Microcontroller work as previously described.

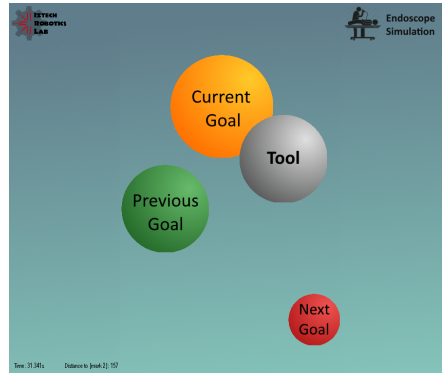

Fig. 5. Screenshot of the training simulator.

The test subjects' goal in each simulation trial is to manipulate the tool to overlap the pre-specified circles one at a time. The metrics used to measure the overall performance of each proposed operation are training time (the time it took to complete a whole run of the simulation), and the number of foot pedal presses per simulation trial (to measure the user's "effort").

For each different mode, we had 10 test subjects perform 10 trials with five unique layouts composed of the same amount of pre-defined circles with different radii that are color-coded to direct the user. The pre-defined circle layout is designed to have the same amount of traveling distance for each run. All users ran the same layouts in the same order allowing us to plot the average improvement in performance over time giving us the learning curve of our system in different modes (Fig.6). We also recoded the number of pedal presses to assess the effort in completing each trial and its improvement after a training period (Fig.7).

Learning Curve

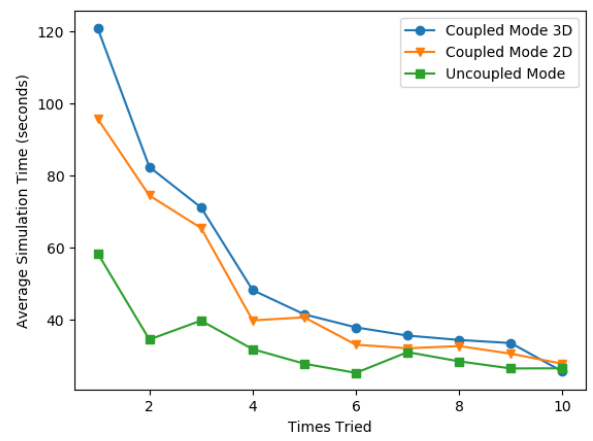

Fig. 6. Plot of average time versus num. of trials.

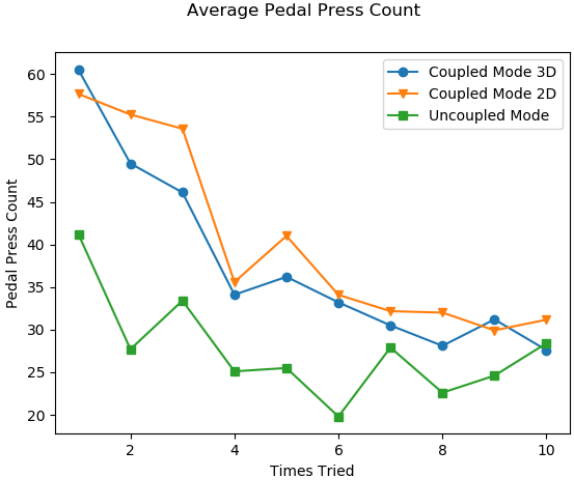

Fig. 7. Plot of average pedal presses per trial. 
It is observed in Fig.6 that in the first 5 trials, coupled mode 2D resulted in slightly shorter completion durations ( $\sim 63 \mathrm{~s}$ average) over 3D ( $\sim 73 \mathrm{~s}$ average) which are longer than the uncoupled mode's ( $\sim 39$ s average). Moreover, two coupled modes have almost the same longer learning curves compared to the uncoupled mode: their completion durations drop to $40 \mathrm{~s}$ limit in the $5^{\text {th }}$ trial whereas the uncoupled mode results drop to $40 \mathrm{~s}$ limit in the $2^{\text {nd }}$ trial. Similar to the decrease in completion durations for each consecutive trial, the average pedal press count shows a decreasing trend in all modes. In the $10^{\text {th }}$ trials, all modes' completion duration converge to $25 \sim 30 \mathrm{~s}$ and pedal press counts converge to $25 \sim 30$ counts.

\section{Conclusion}

In this study, we proposed a teleoperation scheme for an endoscope robot whose 3-DoF is fully actuated. Moreover, we proposed three different operation modes. We evaluated these modes in user studies to assess the usability and user effort. The coupled modes of operation are perceived as more difficult to adapt and requiring more user effort in comparison to the uncoupled mode of operation since they had longer learning curves. Eventually, all modes converge to similar performance levels after a learning process. In the future, an actual endoscope robot will be integrated with this teleoperation scheme.

Acknowledgement This work is supported by The Scientific and Technological Research Council of Turkey via grant number 115E726.

\section{References}

[1] Ballantyne, G. H., And Moll, F. The da Vinci telerobotic surgical system: The virtual operative field and telepresence surgery. Surgical Clinics of North America 83, 6 (2003), 1293-1304.

[2] Dede, M. I. C., MaAroof, O. W., Ates, G., Berker, M., Isikay, I., And HanaliOGLU, S. Unilateral teleoperation design for a robotic endoscopic pituitary surgery system. In New Trends in Medical and Service Robots (Cham, 2018), M. Husty and M. Hofbaur, Eds., Springer International Publishing, pp. 101-115.

[3] Moustris, G. P., Hiridis, S. C., Deliparaschos, K., And Konstantinidis, K. Evolution of autnomous and semi-autnomous robotic surgical systems: a review of the literature. The international journal of medical robotics + computer assisted surgery: MRCAS 7, April (2011), 375-392.

[4] Nathan, C. A. O., Chakradeo, V., Malhotra, K., D’Agostino, H., and PatWARDHAN, R. The voice-controlled robotic assist scope holder AESOP for the endoscopic approach to the sella. Skull Base 16, 3 (2006), 123-132.

[5] Peters, B. S., Armijo, P. R., Krause, C., Choudhury, S. A., And Oleynikov, D. Review of emerging surgical robotic technology. Surgical Endoscopy 32, 4 (Apr 2018), $1636-1655$.

[6] Voros, S., Haber, G. P., Menudet, J. F., Long, J. A., And Cinquin, P. ViKY robotic scope holder: Initial clinical experience and preliminary results using instrument tracking. IEEE/ASME Transactions on Mechatronics 15, 6 (2010), 879-886.

[7] YAŞIR, A., AND KIPER, G. Structural synthesis of 2r1t type mechanisms for minimally invasive surgery applications. In Mechanisms, Transmissions and Applications (Cham, 2018), M. I. C. Dede, M. İtik, E.-C. Lovasz, and G. Kiper, Eds., Springer International Publishing, pp. 31-38. 